\title{
DURAÇAO DO DESEMPREGO E TRANSIÇÕES PARA O EMPREGO FORMAL, A INATIVIDADE E A INFORMALIDADE
}

\author{
MaUrício Reis * \\ Marina Aguas ${ }^{\dagger}$
}

\begin{abstract}
Resumo
Longos períodos de desemprego podem influenciar a probabilidade de o indivíduo encontrar um emprego, assim como podem ter efeitos sobre a decisão desse indivíduo sair do mercado de trabalho. Usando dados da PME (Pesquisa Mensal de Emprego) para o período entre 2006 e 2013, estimamos modelos de duração que consideram transições do desemprego para o emprego, que pode ser formal ou informal, e para a inatividade. De acordo com os resultados, uma determinada característica individual geralmente apresenta efeitos diferenciados dependendo do tipo de transição considerado. Os resultados mostram ainda que as probabilidades de transição para todos os estados analisados aumentam com o tempo de desemprego. Indivíduos há mais tempo no desemprego parecem mais propensos a transitar para um emprego formal ou informal, mas também a deixarem a força de trabalho.
\end{abstract}

Palavras-chave: Duração do desemprego; Emprego; Informalidade; Inatividade.

\begin{abstract}
Long unemployment spells may affect the probability of an unemployed get a job, as well as may be related to transitions out of the labor force. Using data from the PME (Pesquisa Mensal de Emprego) covering the period between 2006 and 2013, we estimated duration models that consider transitions from unemployment to different destinations: i) formal employment, ii) informal employment, and iii) inactivity. According to evidence, individual characteristics usually have heterogeneous effects across the types of transition. The results also show that unemployment duration increases the probability of exit from unemployment to employment (formal or informal) and from unemployment to inactivity.
\end{abstract}

Keywords: Unemployment duration; Employment; Informality; Inactivity.

JEL classification: J60, J64.

DOI: http://dx.doi .org/10.1590/1413-8050/ea416

\footnotetext{
*IPEA.mauricio.reis@ipea.gov.br

† UFF e IBGE.mariffaguas@gmail.com
} 


\section{Introdução}

Uma parcela elevada dos desempregados no Brasil encontra-se afastada do mercado de trabalho há muito tempo. Dados da PME (Pesquisa Mensal de Emprego) mostram que aproximadamente $20 \%$ dos desempregados nas regiões metropolitanas estão nessa situação há um ano ou mais. ${ }^{1}$ Uma consequência direta do desemprego é a incapacidade de o indivíduo auferir renda durante o período de não emprego e, portanto, de contribuir para a renda domiciliar. $\mathrm{O}$ afastamento do mercado de trabalho por muito tempo, no entanto, pode ter ainda outras implicações negativas para o bem-estar dos trabalhadores. Longos períodos de desemprego podem reduzir as oportunidades futuras da pessoa no mercado de trabalho e, com isso, prejudicar sua autoestima, e até sua motivação na busca por emprego.

Diversos artigos na literatura econômica internacional procuram analisar quais os fatores que determinam a saída do indivíduo da condição de desempregado, destacando questões como a dependência da duração e o efeito do seguro-desemprego (Kiefer 1988, Van der Berg 1990, Meyer 1990, McCall 1996, Van der Berg \& Van Ours 1996, 1998) entre outros. Para o Brasil, também podem ser encontrados vários estudos sobre esse tema. Menezes-Filho \& Pichetti (2000) investigam os determinantes da duração do desemprego na região metropolitana de São Paulo e encontram resultados indicando que indivíduos mais velhos e com mais educação tendem a permanecer mais tempo no desemprego. Esses autores mostram também que a probabilidade de saída do desemprego para o emprego inicialmente aumenta e, a partir de seis meses diminui com a duração do desemprego. Penido \& Machado (2002), ampliando a análise para as 6 regiões metropolitanas brasileiras que fazem parte da PME, também encontram que indivíduos mais escolarizados e mais velhos apresentam menor probabilidade de transição do desemprego para o emprego, assim como as mulheres em relação aos homens. Já Abras \& De Felício (2005), diferentemente de Menezes-Filho \& Pichetti (2000), mostram que a probabilidade de saída do desemprego não depende da duração do desemprego, quando são consideradas na análise as heterogeneidades entre os indivíduos. Todos esses artigos para o Brasil, no entanto, consideram um único destino de saída do desemprego, que é a obtenção de um emprego.

Um indivíduo pode deixar a condição de desempregado tanto por ter conseguido um emprego quanto por ter tomado a decisão de deixar a força de trabalho e se tornar inativo. Flinn \& Heckman (1983) mostram que os fatores que determinam as transições para o emprego e para a inatividade são bastante diferentes. Outra questão importante no caso do mercado de trabalho brasileiro é que as saídas do desemprego para o emprego podem envolver uma ocupação formal ou informal.

O objetivo desse artigo é analisar de que maneira a duração do desemprego e as características do trabalhador estão relacionadas com as probabilidades de transição do desemprego para o emprego formal, para o emprego informal e para a inatividade. Na análise empírica, são utilizados dados longitudinais da PME (Pesquisa Mensal de Emprego), que acompanha um mesmo domicílio por um período de até 16 meses, durante o qual são realizadas 8 entrevistas com todos os integrantes do domićlio que tenham 10 anos ou mais. A

\footnotetext{
${ }^{1}$ Para indivíduos com idade entre 21 e 50 anos durante o período de 2006 até 2013, nas seis regiões metropolitanas investigadas pela PME: Belo Horizonte, Porto Alegre, Recife, Rio de Janeiro, Salvador e São Paulo.
} 
abordagem econométrica consiste em estimar, utilizando modelos de duração, a probabilidade de um indivíduo inicialmente desempregado transitar para cada um dos diferentes estados considerados entre a $1^{\text {a }}$ e a $4^{\text {a }}$ entrevista da PME, ou seja, durante um período de 3 meses.

De acordo com os resultados encontrados, algumas características podem influenciar a probabilidade de saída do desemprego de maneira diferenciada, dependendo do destino considerado. O nível de escolaridade, por exemplo, parece influenciar positivamente a probabilidade de transição do desemprego para o emprego formal, mas negativamente as transições para a informalidade e para a inatividade. As mulheres se mostram menos propensas a sair do desemprego para o emprego, seja formal ou informal, mas a probabilidade de transição para a informalidade é maior do que a dos homens, de acordo com os resultados. As evidências mostram que a probabilidade de um trabalhador desempregado obter um emprego, seja formal ou informal, aumenta com a duração do desemprego, e que a probabilidade de transição do desemprego para a inatividade também se torna cada vez maior com o tempo de desemprego.

Esse artigo é composto por quatro seções, além dessa introdução. A seção 2 descreve os dados e apresenta uma análise preliminar, procurando relacionar a duração do desemprego com transições no mercado de trabalho e algumas características dos indivíduos. O método empírico utilizado é descrito na seção 3 e os resultados obtidos são mostrados na seção 4 . Por último, na seção 5, são comentadas as principais conclusões do artigo.

\section{Os dados}

A análise empírica adotada neste artigo utiliza os dados da PME (Pesquisa Mensal de Emprego), realizada pelo IBGE (Instituto Brasileiro de Geografia e Estatística), para o período de janeiro de 2006 até junho de 2013. Essa pesquisa contém várias informações sobre características individuais e do mercado de trabalho de pessoas com 10 anos de idade ou mais, que residem nas 6 principais regiões metropolitanas brasileiras. Pela estrutura longitudinal da PME, é possível acompanhar o mesmo domicílio por um período de até 16 meses. Cada domicílio que entra na pesquisa é entrevistado por quatro meses seguidos, depois permanece 8 meses fora da pesquisa, retornando, então, para mais uma sequência de quatro entrevistas. A cada mês, em torno de $1 / 8$ dos domicílios entrevistados estão participando da pesquisa pela primeira vez.

Como a Tabela A.1 no apêndice mostra, a perda de informações longitudinais sobre os indivíduos na PME é bastante elevada, principalmente entre a $4^{\mathrm{a}}$ e a $5^{\mathrm{a}}$ entrevista. Para a amostra aqui considerada, apenas $42 \%$ dos indivíduos que ingressam na PME permanecem na pesquisa após um ano, quando é realizada a $5^{\text {a }}$ entrevista. Neste estudo, no entanto, são utilizadas apenas as informações para as quatro primeiras entrevistas. Nota-se na Tabela A.1 que entre a $1^{\text {a }}$ e a $4^{\text {a }}$ entrevista a taxa de permanência é de $87 \%$.

A partir dos dados da PME, é possível obter a condição no mercado de trabalho de cada indivíduo, ou seja, se está desempregado, inativo ou empregado. Além disso, a pesquisa oferece informação referente ao período de tempo em que a pessoa passou procurando emprego sem interrupção por mais de duas semanas seguidas, assim como o tempo sem emprego do indivíduo. Essas duas informações são a base para a construção da variável de duração do desem- 
prego, definida como o tempo que uma pessoa sem emprego passou buscando trabalho.

Para os indivíduos empregados, a PME permite identificar a posição na ocupação. Com isso, são consideradas as transições para um emprego formal, caso o indivíduo se torne um empregado com carteira, um empregador, um funcionário público ou um militar, assim como para um emprego informal, no caso de o indivíduo se tornar um empregado sem carteira ou um trabalhador por conta-própria. Também são disponibilizadas na pesquisa informações individuais sobre: anos completos de educação, idade, gênero, raça e região metropolitana de residência. Usando a informação sobre raça, são definidos dois grupos: os negros e pardos são classificados como negros, e os brancos e asiáticos são classificados como brancos. Os indígenas são excluídos da amostra.

A amostra utilizada neste artigo é composta por pessoas com idade entre 21 e 50 anos, que no período da primeira entrevista da PME se encontravam desempregadas na semana de referência, estando nessa situação há pelo menos 7 dias. Ao todo, considerando entradas na pesquisa (1 entrevista do domicílio) durante o período de janeiro de 2006 até fevereiro de 2013, a amostra contém 19.353 indivíduos com dados para as quatro primeiras entrevistas da PME.

A Tabela 1 apresenta as estatísticas descritivas da amostra, para diferentes grupos de trabalhadores definidos de acordo com a duração do desemprego na primeira entrevista de cada indivíduo na PME. A coluna (1) mostra os resultados para as pessoas desempregadas há menos de 12 meses, enquanto a coluna seguinte reporta os resultados para o grupo com desemprego de 1 ano até menos de 2 anos. Na coluna (3), são mostradas as evidências para os desempregados com duração de 2 anos ou mais. Cerca de $80 \%$ dos desempregados se encontram nessa condição há menos de 12 meses. As participações dos grupos de desempregados com durações entre 1 ano e menos de 2 anos e de 2 anos ou mais correspondem a $10 \%$ e $8 \%$ do total de desempregados, respectivamente.

A Tabela 1 mostra que as médias para os anos completos de escolaridade e para a idade são bastante parecidas entre as colunas. Já as proporções de mulheres, de negros e, principalmente, de indivíduos que nunca trabalharam anteriormente, aumentam com a categoria de duração do desemprego. Entre os indivíduos com 2 anos ou mais de desemprego, 67\% são mulheres e 58\% são classificados como negros. Já entre aqueles com menos de 12 meses de desemprego, as participações das mulheres e dos negros correspondem a $58 \%$ e $52 \%$, respectivamente. Nota-se também que apenas $7,5 \%$ dos indivíduos desempregados há menos de um ano nunca tiveram trabalho anteriormente. A participação desse grupo aumenta para 16\% entre aqueles com duração do desemprego entre 1 e 2 anos, enquanto entre as pessoas com dois anos ou mais de desemprego $20 \%$ nunca trabalharam anteriormente, o que sugere uma dificuldade maior para um trabalhador conseguir o seu primeiro emprego.

A Tabela 1 também reporta a proporção de trabalhadores que transitaram para a inatividade depois de 3 meses da primeira entrevista. Percebe-se que esse tipo de transição aumenta com a duração do desemprego. Entre os desempregados há menos de 12 meses, a proporção que saiu da força de trabalho corresponde a 31,5\%. Já entre os desempregados há 2 anos ou mais, $40 \%$ se moveram do desemprego para a inatividade 3 meses depois. Com relação ás transições do desemprego para o emprego nos 3 meses seguintes, cabe 
Tabela 1: Estatísticas descritivas

\begin{tabular}{|c|c|c|c|}
\hline & \multicolumn{3}{|c|}{ Duração do desemprego } \\
\hline & Até 1 ano & $\begin{array}{l}\text { De } 1 \text { ano até menos } \\
\text { de } 2 \text { anos }\end{array}$ & 2 anos ou mais \\
\hline Anos de escolaridade & 9,8 & 9,7 & 9,4 \\
\hline Idade & 31,3 & 30,8 & 32,6 \\
\hline Mulher (\%) & 57,6 & 62,9 & 66,6 \\
\hline Negro $(\%)$ & 52,2 & 54,5 & 57,8 \\
\hline Nunca trabalhou (\%) & 7,5 & 15,8 & 19,5 \\
\hline \multicolumn{4}{|c|}{ Transições entre a 1a. e a 4a. entrevista } \\
\hline Desemprego =>Inatividade $(\%)$ & 31,5 & 35 & 40 \\
\hline Desemprego => Emprego $(\%)$ & 29,4 & 17,8 & 15,9 \\
\hline $\begin{array}{l}\text { Emprego formal entre os que transitaram } \\
\text { do desemprego para o emprego }(\%)\end{array}$ & 44,9 & 34,9 & 35,6 \\
\hline Desemprego => Emprego formal $(\%)$ & & 6,2 & 5,7 \\
\hline Desemprego => Emprego informal $(\%)$ & 16,2 & 11,6 & 10,2 \\
\hline Observações & 15.816 & 1.894 & 1.643 \\
\hline \multicolumn{4}{|c|}{$\begin{array}{l}\text { Fonte: PME 2006-2013. } \\
\text { A amostra incluí apenas trabalhadores desempregados no período da primeira entrevista. } \\
\text { Transições para a inatividade e o emprego são referentes ao intervalo de } 3 \text { meses após a } \\
\text { primeira entrevista. }\end{array}$} \\
\hline
\end{tabular}

destacar que a proporção de trabalhadores que experimentaram esse tipo de mobilidade diminui com a duração do desemprego. Dos indivíduos há menos tempo desempregados, $29 \%$ conseguiram emprego 3 meses depois, enquanto entre os desempregados há 2 anos ou mais essa proporção foi de apenas $16 \%$. Nota-se também que entre os trabalhadores que transitaram para o emprego, $45 \%$ se moveram para o setor formal, quando considerados apenas aqueles com menos de um ano de desemprego. Para os trabalhadores com 2 anos ou mais de duração do desemprego, entretanto, apenas 36\% transitaram para o setor formal, entre os que conseguiram emprego.

De acordo com a análise descritiva apresentada na Tabela 1, portanto, a proporção de trabalhadores que transitam do desemprego para a inatividade é maior entre os que estão desempregados há mais tempo. Deve-se ressaltar também que a proporção de trabalhadores que conseguem emprego diminui bastante com o tempo de desocupação e, normalmente, essas transições passam a ter como destino o setor informal. Algumas características individuais dos trabalhadores, como idade e escolaridade, no entanto, são em média parecidas entre os grupos de duração do desemprego, enquanto negros e mulheres parecem ter uma representatividade maior entre os desempregados de mais longo prazo, assim como aqueles que nunca trabalharam.

\section{Método empírico}

Essa seção descreve brevemente a metodologia usada nesse artigo, que consiste em estimar modelos de duração em que a saída do desemprego considera diferentes estados, conhecidos como modelos de riscos competitivos (ver, por exemplo, Cameron e Trivedi, 2005 e Addison e Portugal, 2003). Para isso, considere a função de risco, que oferece a probabilidade instantânea de o indivíduo deixar o desemprego em um determinado período $t$, dado que perma- 
neceu desempregado até $t$. A função de risco pode ser representada como:

$$
h(t, x)=\lim _{\mathrm{dt} \rightarrow 0} \frac{P(t \leq T<t+\mathrm{dt} / T \geq t, x)}{\mathrm{dt}}=\frac{f(t, x)}{1-F(t, x)}=\frac{f(t, x)}{S(t, x)}
$$

Onde $x$ é um conjunto de covariadas, $F(t, x)$ é a função distribuição acumulada, e $f(t, x)$ é a função densidade de probabilidade, onde $f(t, x)=\mathrm{dF}(t, x) / \mathrm{dt}$.

A função sobrevivência, representada por $S(t, x)$, fornece a probabilidade de que a duração do desemprego seja maior ou igual a $t$, onde $S(t, x)=1-$ $F(t, x)$. A função de risco integrada é definida por $\Lambda(t, x)=\int_{0}^{t} h(s, x)$ ds, e está relacionada da seguinte maneira com a função sobrevivência: $S(t, x)=e^{-\Lambda(t, x)}$.

$\mathrm{Na}$ análise adotada nesse artigo, são considerados três diferentes destinos possíveis de saída do desemprego: i) emprego formal, ii) emprego informal, e iii) inatividade. Dessa forma, a função de risco para o destino $j$ pode ser definida como:

$$
h_{j}(t, x)=\lim _{\mathrm{dt} \rightarrow 0} \frac{P(t \leq T<t+\mathrm{dt} / T \geq t, \mathrm{x}, J=j)}{\mathrm{dt}}, j=1,2,3 .
$$

A equação (2) oferece a probabilidade instantânea de que um indivíduo com as características representadas pelo vetor $x$ deixe o desemprego para o destino $\mathrm{j}$, dado que permaneceu desempregado até o período $t$. A partir da equação (2), podem ser definidas a função de risco agregada, $h(t, x)=\sum_{j=1}^{3} h_{j}(t, x)$, e a seguinte função sobrevivência: $S(t, x)=\prod_{j=1}^{3} S^{j}(t, x)$, onde $S^{j}(t, x)=e^{-\Lambda^{j}(t, x)}$, e $\Lambda^{j}(t, x)=\int_{0}^{t} h_{j}(s, x) \mathrm{ds}$

Uma hipótese importante que deve ser mencionada é que se supõe nessa abordagem que os riscos de saída para cada um dos destinos considerados são independentes com duração contínua.

Diferentes abordagens são utilizadas nesse artigo para analisar as transições do desemprego para o emprego formal, a inatividade e a informalidade. Primeiramente, são apresentados resultados para a função de incidência acumulada, em que as saídas do desemprego para o destino $\mathrm{j}$ podem ser estimadas da seguinte maneira:

$$
\hat{I}_{j}\left(t_{m}\right)=\sum_{t=1}^{m} \hat{S}\left(t_{m}\right) \frac{d_{\mathrm{jm}}}{n_{m}}, m=1,2, \ldots . M-1, .
$$

onde $\hat{S}\left(t_{m}\right)=\prod_{t=1}^{m} \frac{n_{t}-d_{t}}{n_{t}}$ é o estimador de Kaplan-Meier para a função sobrevivência, considerando saídas de todos os tipos, e $\frac{d_{j m}}{n_{m}}$ é um estimador da função de risco para saídas do tipo j, sendo $d_{j m}$ o número de transições do desemprego para o destino j no período $t_{\mathrm{m}}$ e $n_{m}$ é o número de indivíduos em risco no período $t_{m-1}$.

As transições também são analisadas utilizando um modelo paramétrico, em que a função de risco é representada por uma Weibull. Nesse caso, para um indivíduo com características $x_{i}$, temos: 


$$
h_{j}\left(t, x_{i}\right)=\alpha_{j} \lambda_{\mathrm{ji}}^{\alpha} t^{\alpha-1}, \lambda_{\mathrm{ji}}=e^{x_{\mathrm{ji}}^{\prime} \beta_{j}}, j=1,2,3 .
$$

O parâmetro $\alpha_{j}$ representa a dependência da duração. Quando $\alpha_{j}>1$, a função é crescente, indicando uma dependência positiva da duração, enquanto para $\alpha_{j}<1$ a dependência da duração é negativa. Quando $\alpha_{j}=1$ a duração do desemprego não influencia a probabilidade de saída desse estado para o destino j. Os parâmetros do modelo são estimados pelo método de máxima verossimilhança a partir dos dados da PME, e as seguintes variáveis fazem parte de $x$ : escolaridade, idade, variáveis dummy para mulheres, negros e indivíduos que nunca trabalharam anteriormente, e dummies para os anos e as regiões metropolitanas.

Os resultados também são estimados usando um modelo semiparamétrico, que permite mais flexibilidade para a função de risco. No modelo de Cox de riscos proporcionais, a função de risco é definida como:

$$
h_{j}\left(t, x_{i}\right)=h_{j 0}(t) \varphi\left(x_{i}, \beta_{j}\right)
$$

Nesse caso, a função base de risco, $h_{j 0}(t)$, é estimada de maneira não paramétrica (Cox 1972,1975), e $\varphi\left(x_{i}, \beta_{j}\right)$ é representada por uma função exponencial.

\section{Resultados}

Na figura 1, a função de incidência acumulada mostra saídas mais frequentes para a inatividade do que para o emprego, seja formal ou informal, com o tempo de desemprego. Nota-se também que a diferença entre as saídas do desemprego para a inatividade em relação ao emprego tende a se ampliar à medida que aumenta o tempo de permanência no desemprego.

A probabilidade estimada de saída do desemprego para a inatividade no período de até 4 trimestres é igual a 32\%. Para transições do desemprego para o emprego formal e para o emprego informal durante esse mesmo período, as probabilidades estimadas são iguais a $15 \%$ e $17 \%$, respectivamente. Considerando o período de 20 trimestres, a probabilidade de transição para a inatividade é estimada em $50 \%$, enquanto as probabilidades de saída para o emprego formal e para o emprego informal são estimadas em $18 \%$ e $23 \%$, respectivamente.

Na figura A1 do apêndice, são apresentados os resultados estimados para a função de incidência acumulada separadamente por gênero, idade e escolaridade. As diferenças por gênero são acentuadas, com a proporção de mulheres que transitam do desemprego para a inatividade aumentando bastante com a duração do desemprego, enquanto entre os homens as saídas do desemprego para o emprego são mais intensas do que entre as mulheres. Já as diferenças por idade, representadas no painel (b), são pequenas. De acordo com os resultados por escolaridade, porém, percebe-se que transições do desemprego para o emprego formal são mais frequentes entre os mais escolarizados, e que os indivíduos com níveis educacionais mais baixos, quando transitam para o emprego, o fazem com uma intensidade bem maior para o setor informal.

Na Tabela 2, são apresentados os resultados estimados para a probabilidade de saída do desemprego usando modelos paramétricos, em que a função 


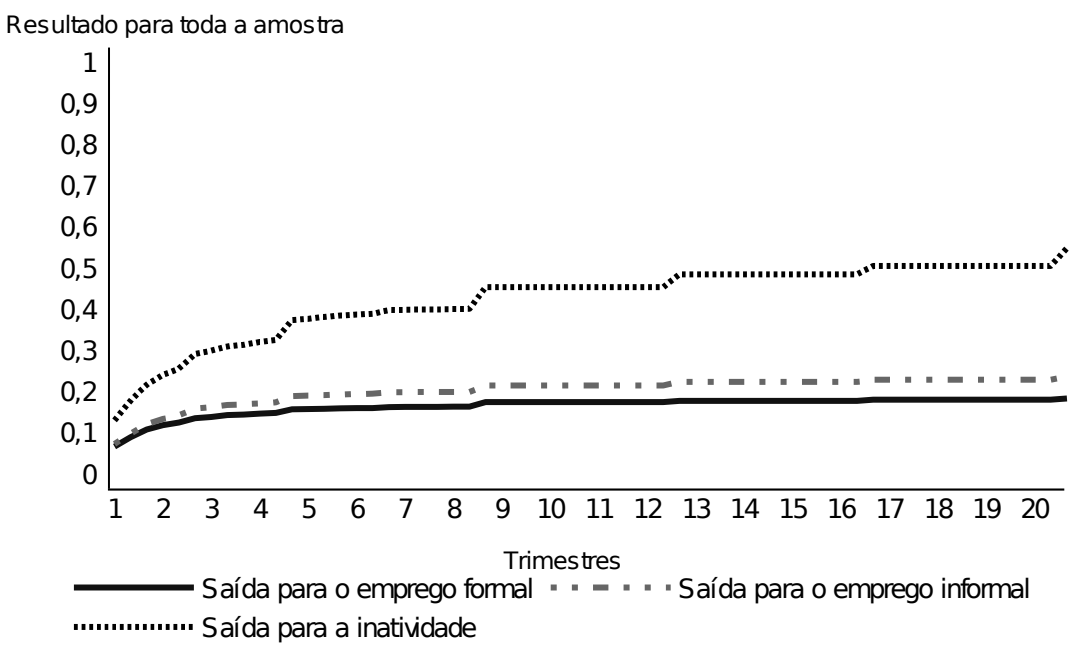

Figura 1: Função de incidência calculada

de risco é representada por uma distribuição Weibull. São consideradas transições para três estados: i) emprego formal, ii) emprego informal e iii) inatividade, em um intervalo de 3 meses. ${ }^{2}$ Nas colunas (1), (2) e (3), são mostrados os resultados sem heterogeneidades não observadas entre os indivíduos, enquanto as colunas (4), (5) e (6) mostram os resultados que incluem as heterogeneidades não observadas na análise. ${ }^{3}$

Os resultados mostram que a probabilidade de saída do desemprego para um emprego formal é maior para os indivíduos mais escolarizados. Níveis mais altos de escolaridade, por outro lado, parecem diminuir as probabilidades de saída do desemprego para a informalidade e para a inatividade. Para cada ano adicional de estudo, o risco de saída para o emprego formal aumenta em $2,1 \%$ [exp $(0,021)-1]$ na coluna $(1)$, e em $4 \%$ [exp(0,039)-1] na coluna (2). O risco de saída para o emprego informal diminui entre 4\% e 7\%, enquanto o risco de saída para a inatividade diminui cerca de $1 \%$, para cada ano de estudo a mais, de acordo com os resultados estimados.

Menezes-Filho \& Pichetti (2000) encontram uma relação negativa entre anos de estudo e probabilidade de saída do desemprego para o emprego, que os autores interpretam como sendo consequência de um salário de reserva mais alto para os mais escolarizados, que são mais seletivos no processo de busca. Esse mesmo argumento pode ser usado como explicação para as evidências reportadas na Tabela 2. Enquanto os menos escolarizados transitam do desemprego para um emprego informal, aqueles com maior nível educacional parecem preferir aguardar uma proposta mais atrativa no setor formal. Para os menos escolarizados, a falta de oportunidades também parece levar os indivíduos a desistir de procurar emprego.

\footnotetext{
${ }^{2}$ Os resultados são semelhantes considerando transições do desemprego para esses outros estados no período de um mês, como mostrado no apêndice.

${ }^{3} \mathrm{~A}$ heterogeneidade não observada é representada por um termo multiplicativo $\left(v_{j}\right)$ associado a cada função de risco: $h_{j}\left(t, x_{i}, v\right)=\alpha_{j} \lambda_{\mathrm{ji}}^{\alpha} t^{\alpha-1} v_{j}$, onde $\lambda_{\mathrm{ji}}=e^{x_{\mathrm{ji}}^{\prime} \beta_{j}}$. Supõe-se que esse termo multiplicativo tem distribuição gaussiana inversa.
} 
Tabela 2: Modelo paramétrico para duração do desemprego (função Weilbull)

\begin{tabular}{|c|c|c|c|c|c|c|}
\hline \multicolumn{7}{|c|}{ Transições do desemprego para: emprego formal, informal e inatividade } \\
\hline & \multicolumn{3}{|c|}{ Sem heterogeneidade } & \multicolumn{3}{|c|}{ Com heterogeneidade } \\
\hline & $\begin{array}{l}\text { Formal } \\
\text { (1) }\end{array}$ & $\begin{array}{c}\text { Informal } \\
(2)\end{array}$ & $\begin{array}{c}\text { Inatividade } \\
\text { (3) }\end{array}$ & $\begin{array}{l}\text { Formal } \\
\qquad(4)\end{array}$ & $\begin{array}{l}\text { Informal } \\
\text { (5) }\end{array}$ & $\begin{array}{c}\text { Inatividade } \\
(6)\end{array}$ \\
\hline Escolaridade & $\begin{array}{l}0,021^{* * *} \\
(0,006)\end{array}$ & $\begin{array}{c}-0,045^{* * *} \\
(0,006)\end{array}$ & $\begin{array}{c}-0,009^{* *} \\
(0,004)\end{array}$ & $\begin{array}{l}0,039^{* * *} \\
(0,009)\end{array}$ & $\begin{array}{c}-0,069^{* * *} \\
(0,008)\end{array}$ & $\begin{array}{c}-0,017^{* * *} \\
(0,006)\end{array}$ \\
\hline Idade & $\begin{array}{c}-0,018^{* * *} \\
(0,003)\end{array}$ & $\begin{array}{l}-0,002 \\
(0,002)\end{array}$ & $\begin{array}{c}-0,009^{* * *} \\
(0,002)\end{array}$ & $\begin{array}{c}-0,023^{* * *} \\
(0,004)\end{array}$ & $\begin{array}{c}0 \\
(0,004)\end{array}$ & $\begin{array}{c}-0,012^{* * *} \\
(0,003)\end{array}$ \\
\hline Mulher & $\begin{array}{c}-0,543^{* * *} \\
(0,042)\end{array}$ & $\begin{array}{c}-0,491^{* * *} \\
(0,040)\end{array}$ & $\begin{array}{l}0,243^{* * *} \\
(0,030)\end{array}$ & $\begin{array}{c}-0,839^{* * *} \\
(0,061)\end{array}$ & $\begin{array}{c}-0,713^{* * *} \\
(0,055)\end{array}$ & $\begin{array}{l}0,382^{* * *} \\
(0,042)\end{array}$ \\
\hline Negro & $\begin{array}{l}0,121 \\
(0,048)\end{array}$ & $\begin{array}{l}0,089 \\
(0,043)\end{array}$ & $\begin{array}{r}-0,030 \\
(0,031)\end{array}$ & $\begin{array}{l}0,145 \\
(0,069)\end{array}$ & $\begin{array}{l}0,107 \\
(0,063)\end{array}$ & $\begin{array}{r}-0,053 \\
(0,046)\end{array}$ \\
\hline Nunca trabalhou & $\begin{array}{c}-0,995^{* * *} \\
(0,093)\end{array}$ & $\begin{array}{c}-0,601^{* * *} \\
(0,077)\end{array}$ & $\begin{array}{c}-0,224^{* * *} \\
(0,043)\end{array}$ & $\begin{array}{c}-1,436^{* * *} \\
(0,133)\end{array}$ & $\begin{array}{c}-0,888^{* * *} \\
(0,113)\end{array}$ & $\begin{array}{c}-0,324^{* * *} \\
(0,068)\end{array}$ \\
\hline Parâmetro $\alpha$ & $\begin{array}{l}0,852 \\
(0,007)\end{array}$ & $\begin{array}{l}0,847 \\
(0,008)\end{array}$ & $\begin{array}{l}0,878 \\
(0,009)\end{array}$ & $\begin{array}{l}1,327 \\
(0,015)\end{array}$ & $\begin{array}{l}1,344 \\
(0,014)\end{array}$ & $\begin{array}{l}1,427 \\
(0,011)\end{array}$ \\
\hline Parâmetro $\sigma 2$ & & & & $\begin{array}{r}12,541 \\
(0.685) \\
\end{array}$ & $\begin{array}{r}12,804 \\
(0.519) \\
\end{array}$ & $\begin{array}{l}8,243 \\
(0.215) \\
\end{array}$ \\
\hline Observações & 19.353 & 19.353 & 19.353 & 19.353 & 19.353 & 19.353 \\
\hline
\end{tabular}

Notas: os erros-padrão são apresentados entre parênteses.

Todas as regressões incluem dummies para as regiões metropolitanas e para os anos.

* significativo para o nível de $10 \%$.

** significativo para o nível de $5 \%$.

${ }^{* * *}$ significativo para o nível de $1 \%$. 
Nas colunas (1) e (4), nota-se que os indivíduos mais velhos são menos propensos a sair do desemprego para um emprego formal em comparação com os trabalhadores mais jovens. Além disso, a Tabela 2 mostra que a probabilidade de transitar do desemprego para a inatividade também é menor para os trabalhadores mais velhos. Para cada ano de idade a mais, o risco de saída para o emprego formal diminui $2 \%$, enquanto o risco de saída para a inatividade diminui $1 \%$. Para as transições do desemprego para a informalidade, nas colunas (2) e (5), as diferenças por idade não são estatisticamente significativas.

Para as mulheres, a probabilidade de transição do desemprego para o emprego, seja formal ou informal, é menor do que a dos homens. O risco de saída para o emprego formal é $42 \%$ menor para as mulheres na coluna (1), enquanto na coluna (4) o diferencial em relação aos homens é estimado em -57\%. Os riscos estimados de saída para o emprego informal são entre $39 \%$ e $50 \%$ menores para as mulheres. Nota-se também, que as mulheres, quando desempregadas, apresentam uma probabilidade maior de deixarem o mercado de trabalho do que os homens. Na coluna (3), o risco e saída para a inatividade é $28 \%$ maior para as mulheres, enquanto na coluna (6) essa diferença é de $47 \%$.

Ainda de acordo com os resultados da Tabela 2, os negros apresentam maiores probabilidades de transição para empregos formais e informais do que os brancos. O risco de saída para o primeiro destino é cerca de $15 \%$ maior para os negros, que também apresentam um risco de saída para a informalidade $10 \%$ maior do que os brancos, de acordo com as evidências. Para as probabilidades de transição do desemprego para a inatividade, as diferenças entre negros e brancos não são estatisticamente significativas.

Indivíduos que nunca trabalharam anteriormente apresentam probabilidades mais baixas de obterem empregos formais e informais do que indivíduos desempregados que já tiveram algum emprego anterior. No caso do risco de saída para o emprego formal, o diferencial estimado dos indivíduos que nunca trabalharam em relação aos que já trabalharam anteriormente se situa entre $-63 \%$ e $-76 \%$. Já o risco de saída para o emprego informal se mostra entre $45 \%$ e $59 \%$ menor para os que nunca trabalharam. Esses resultados são semelhantes aos encontrados por Menezes-Filho \& Pichetti (2000) para a região metropolitana de São Paulo, e indicam certa dificuldade para se obter o primeiro emprego. ${ }^{4}$ A Tabela 2 mostra também que o fato de nunca ter trabalhado anteriormente diminui a probabilidade de o indivíduo desistir de procurar emprego e se tornar inativo. Na coluna (6), o risco de uma pessoa que nunca trabalhou antes sair do desemprego para a inatividade é $28 \%$ menor do que o de um indivíduo que já trabalhou.

As colunas (1), (2) e (3) da Tabela 2, que não consideram as heterogeneidades não observadas, mostram que transições para o emprego formal, o emprego informal e para a inatividade apresentam dependência negativa da duração. Ou seja, quanto maior o tempo de desemprego, menor a probabilidade de sair desse estado e transitar para cada um desses destinos considerados.

Porém, quando são consideradas as heterogeneidades não observadas, nas colunas (4), (5) e (6), os resultados se tornam bem diferentes. Nota-se também, que a estimativa para a variância $\left(\sigma^{2}\right)$ é significativa, indicando a presença de heterogeneidade não observada. As evidências que indicam a presença de dependência negativa da duração, nas colunas (1), (2) e (3), parecem decorren-

\footnotetext{
${ }^{4}$ Entre esses indivíduos que declararam nunca terem trabalhado anteriormente, $2 / 3$ são jovens com idade entre 21 e 25 anos.
} 
tes da agregação de indivíduos com taxas de saída do desemprego diferentes. Considerando as heterogeneidades não observadas, os indivíduos desempregados há mais tempo apresentam maior probabilidade de saída para o emprego formal, assim como para o emprego informal. Nota-se também que uma duração maior do desemprego está associada com uma probabilidade mais elevada de transição para a inatividade. De acordo com esse resultado, portanto, indivíduos há muito tempo desempregados parecem desistir de procurar emprego por desalento.

Evidências internacionais mostram estimativas bastante heterogêneas entre os países para a relação entre duração do desemprego e probabilidade de saída desse estado, como pode ser verificado na série de estudos analisados por Machin \& Manning (1999). Para os estudos que consideram múltiplos destinos de saída do desemprego, os resultados para a Áustria (Winter-Ebner 1998) e para a Suécia (Edin 1989) mostram uma dependência positiva da duração para transições do desemprego para o emprego. Já os resultados para Portugal (Dias 1997) e Espanha (Alba 1999) mostram uma dependência da duração negativa, enquanto para a Alemanha, Steiner (1997) encontra dependência da duração positiva para os homens e negativa para as mulheres. Quanto às transições do desemprego para a inatividade, não são apresentadas evidências de dependência da duração negativa, e resultados indicando dependência da duração positiva são encontrados nos estudos acima para Áustria, Alemanha, Espanha e Suécia.

Tabela 3: Modelo Cox para duração do desemprego

\begin{tabular}{lccc}
\hline \multicolumn{3}{l}{ Transições do desemprego para: emprego formal, informal e inatividade } \\
\hline & Formal & Informal & Inatividade \\
& $(1)$ & $(2)$ & $(3)$ \\
\hline Escolaridade & $0,023^{* * *}$ & $-0,045^{* * *}$ & $-0,009^{* *}$ \\
& $(0,006)$ & $(0,005)$ & $(0,004)$ \\
Idade & $-0,012^{* * *}$ & 0,003 & $-0,005^{* * *}$ \\
& $(0,002)$ & $(0,002)$ & $(0,002)$ \\
Mulher & $-0,496^{* * *}$ & $-0,449^{* * *}$ & $0,278^{* * *}$ \\
& $(0,038)$ & $(0,034)$ & $(0,025)$ \\
Negro & $0,100^{* *}$ & $0,069^{*}$ & $-0,037$ \\
& $(0,044)$ & $(0,039)$ & $(0,027)$ \\
Nunca trabalhou & $-0,802^{* * *}$ & $-0,436^{* * *}$ & $-0,110^{* * *}$ \\
& $(0,088)$ & $(0,069)$ & $(0,038)$ \\
Observações & 19353 & 19353 & 19353 \\
\hline Todas as regressões incluem dummies para as regiões metropolitanas e para os anos. \\
*significativo para o nível de 10\%. \\
**significativo para o nível de 5\%. \\
***significativo para o nível de $1 \%$.
\end{tabular}

A Tabela 3 apresenta os resultados estimados usando o modelo semiparamétrico de Cox. Para cada uma das variáveis explicativas, a relação entre a probabilidade de o indivíduo deixar o desemprego para cada um dos três destinos considerados é semelhante à reportada na Tabela 2.

A Figura 2 descreve os resultados estimados para as funções de risco acumuladas, de acordo com o modelo Cox. Deve-se enfatizar que esses resultados refletem não apenas a dependência da duração, mas também efeitos associados com a heterogeneidade não observada. Percebe-se que as transições para 
o emprego formal são as menos frequentes entre os três destinos considerados. Além disso, as saídas do desemprego se mostram mais intensas quando tem como destino a inatividade.

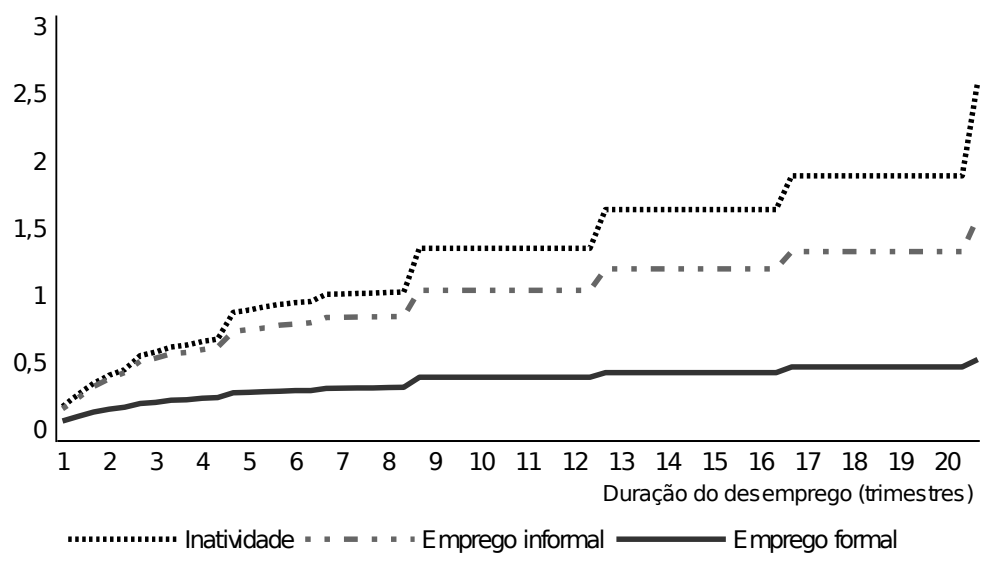

Figura 2: Estimador de Cox-função de risco acumulada

\section{Conclusões}

Investigamos neste artigo de que maneira a duração do desemprego e as características do indivíduo influenciam as suas probabilidades de transição da condição de desempregado para as condições de empregado formal ou informal, ou de inativo. Nessa análise, são usados os microdados da PME para o período de janeiro de 2006 até junho de 2013, já que a PME permite que seja observada a condição de um mesmo indivíduo no mercado de trabalho em diferentes períodos de tempo e, com isso, identificar transições do desemprego para outros estados.

De acordo com os resultados estimados, a escolaridade aumenta a probabilidade de transição para o emprego formal e diminui as probabilidades de saída do desemprego para o emprego informal e para a inatividade. Transições para o emprego formal e a inatividade parecem mais frequentes para os indivíduos mais novos, enquanto os que nunca trabalharam anteriormente se mostram menos propensos a deixar o desemprego para qualquer um dos estados considerados. As probabilidades de transição para empregos formais e informais são menores para as mulheres, que também apresentam uma probabilidade maior de transitarem do desemprego para a inatividade do que os homens. Portanto, as características individuais e o fato de já ter trabalhado anteriormente parecem influenciar a trajetória do desempregado até a obtenção de um emprego ou até a decisão de sair do mercado de trabalho.

As evidências mostram que quanto maior a duração do desemprego, maiores as probabilidades de transição para o emprego formal e para o emprego informal, quando consideradas as heterogeneidades não observadas entre os indivíduos. Esse comportamento pode estar associado a um processo de redução no salário de reserva ao longo do período de desemprego, fazendo com 
que o indivíduo passe a aceitar ofertas de emprego que não aceitaria anteriormente. Os resultados também indicam que o desemprego de longo prazo aumenta a probabilidade de saída da força de trabalho. Portanto, o desestímulo para continuar a busca por emprego parece levar os trabalhadores desempregados há muito tempo a se retirarem do mercado de trabalho por desalento.

\section{Agradecimentos}

Os autores agradecem pelos comentários de um parecerista anônimo. Mauricio Reis agradece o apoio financeiro do CNPQ.

\section{Referências Bibliográficas}

Abras, A. \& De Felício, F. (2005), 'Duração e taxa de saída do desemprego: evidência de ausência de dependência na duração para as regiões metropolitanas no brasil (1984-2000)', XXXIII Encontro Nacional de Economia.

Alba, A. (1999), 'Explaining the transitions out of unemployment in spain: the effect of unemployment insurance', Applied Economics 31(2), 183-193.

Cox, D. (1972), 'Regression models and life tables', Journal of the Royal Statistical Society 32, 187-220.

Cox, D. (1975), 'Partial likelihood', Biometrika 62(2), 269-76.

Dias, M. (1997), 'A study on labour market mobility', Mimeo.

Edin, P. (1989), 'Unemployment duration and competing risk: evidence from sweden', Scandinavian Journal of Economics 91(4), 639-53.

Flinn, C. \& Heckman, J. (1983), 'Are unemployment and out of the labor force behaviorally distinct labor market states?', Journal of Labor Economics 1(1), 28-42.

Kiefer, N. (1988), 'Economic duration data and hazard functions". journal of economic literature', Journal of Economic Literature 26(2), 646-79.

Machin, S. \& Manning, A. (1999), Longterm unemployment in europe, in D. Ashenfelter, O ; Card, ed., 'Handbook of Labor Economics', Vol. 3c, Elsevier.

McCall, B. (1996), 'Unemployment insurance rules, joblessness, and parttime work', Econometrica 64(3), 647-82.

Menezes-Filho \& Pichetti (2000), 'Os determinantes da duração do desemprego no brasil', Pesquisa e Planejamento Econômico 30(1), 23-48.

Meyer, B. (1990), 'Unemployment insurance and unemployment spells', Econometrica 58(4), 757-82.

Penido, M. \& Machado, A. (2002), 'Desemprego: evidência da duração no brasil metropolitano', CEDEPLAR/UFMG: Texto para Discussão n. 83.

Steiner, V. (1997), 'Extended benefit entitlement periods and the duration of unemployment in west germany', ZEW Discussion Papers 97-14. 
Van der Berg, G. (1990), 'Nonstationarity in job search theoty', Review of Economics Studies 57(2), 255-77.

Van der Berg, G. \& Van Ours, J. (1996), 'Unemployment dynamics and duration dependence', Journal of Labor Economics 14(1), 100-125.

Van der Berg, G. \& Van Ours, J. (1998), 'On the detection of state dependence using aggregate outflow data: comments on previous studies', The Economic Journal 108(450), 1422-30.

Winter-Ebner (1998), 'Potential unemployment benefit duration and spell length: lessons from a quasi-experiment in austria', Oxford Bulletin of Economics and Statistics 60, 3-46.

\section{Apêndice A}

Tabela A.1: Descriptive statistics

\begin{tabular}{lcc}
\hline & Número de observações & $\begin{array}{c}\text { Parcela de observações em relação } \\
\text { à primeira entrevista }(\%)\end{array}$ \\
\hline Entrevista 1 & 18,544 & 100 \\
Entrevista 2 & 17,566 & 94,73 \\
Entrevista 3 & 16,859 & 90,91 \\
Entrevista 4 & 16,169 & 87,19 \\
Entrevista 5 & 7,755 & 41,82 \\
Entrevista 6 & 7,562 & 40,78 \\
Entrevista 7 & 7,357 & 39,67 \\
Entrevista 8 & 7,072 & 38,14 \\
\hline
\end{tabular}

Fonte: PME 2006-2011.

Amostra: trabalhadores desempregados no período da primeira entrevista, com idade entre 21 e 50 anos. 
Tabela A.2: Modelo paramétrico para duração do desemprego (função Weilbull)

\begin{tabular}{|c|c|c|c|c|c|c|}
\hline & \multicolumn{3}{|c|}{ Sem heterogeneidade } & \multicolumn{3}{|c|}{ Com heterogeneidade } \\
\hline & Formal & Informal & Inatividade & Formal & Informal & Inatividade \\
\hline & (1) & $(2)$ & (3) & (4) & (5) & (6) \\
\hline Escolaridade & $\begin{array}{l}0,02^{* * *} \\
(0,009)\end{array}$ & $\begin{array}{c}-0,053^{* * *} \\
(0,007)\end{array}$ & $\begin{array}{c}-0,011^{* * *} \\
(0,004)\end{array}$ & $\begin{array}{l}0,04^{* * *} \\
(0,013)\end{array}$ & $\begin{array}{c}-0,079^{* * *} \\
(0,010)\end{array}$ & $\begin{array}{c}-0,019^{* * *} \\
(0,006)\end{array}$ \\
\hline Idade & $\begin{array}{c}-0,015^{* * *} \\
(0,004)\end{array}$ & $\begin{array}{r}-0,003 \\
(0,003)\end{array}$ & $\begin{array}{c}-0,01^{* * *} \\
(0,002)\end{array}$ & $\begin{array}{c}-0,019^{* * *} \\
(0,006)\end{array}$ & $\begin{array}{r}-0,002 \\
(0,004)\end{array}$ & $\begin{array}{c}-0,014^{* * *} \\
(0,003)\end{array}$ \\
\hline Mulher & $\begin{array}{c}-0,513^{* * *} \\
(0,059)\end{array}$ & $\begin{array}{c}-0,542^{* * *} \\
(0,046)\end{array}$ & $\begin{array}{l}0,105^{* * *} \\
(0,028)\end{array}$ & $\begin{array}{c}-0,776^{* * *} \\
(0,087)\end{array}$ & $\begin{array}{c}-0,782^{* * *} \\
(0,066)\end{array}$ & $\begin{array}{l}0,159^{* * *} \\
(0,042)\end{array}$ \\
\hline Negro & $\begin{array}{l}0,205^{* * *} \\
(0,069)\end{array}$ & $\begin{array}{l}0,029 \\
(0,052)\end{array}$ & $\begin{array}{r}-0,026 \\
(0,031)\end{array}$ & $\begin{array}{l}0,257^{* *} \\
(0,100)\end{array}$ & $\begin{array}{l}0,018 \\
(0,075)\end{array}$ & $\begin{array}{r}-0,061 \\
(0,046)\end{array}$ \\
\hline Nunca trabalhou & $\begin{array}{c}-0,87^{* * *} \\
(0,128)\end{array}$ & $\begin{array}{c}-0,721^{* * *} \\
(0,097)\end{array}$ & $\begin{array}{c}-0,224^{* * *} \\
(0,044)\end{array}$ & $\begin{array}{c}-1,256^{* * *} \\
(0,184)\end{array}$ & $\begin{array}{c}-1,047^{* * *} \\
(0,139)\end{array}$ & $\begin{array}{c}-0,312^{* * *} \\
(0,067)\end{array}$ \\
\hline Parâmetro $\alpha$ & $\begin{array}{l}0,864 \\
(0,012)\end{array}$ & $\begin{array}{l}0,857 \\
(0,010)\end{array}$ & $\begin{array}{l}0,871 \\
(0,006)\end{array}$ & $\begin{array}{l}1,333 \\
(0,023)\end{array}$ & $\begin{array}{l}1,331 \\
(0,017)\end{array}$ & $\begin{array}{l}1,408 \\
(0,011)\end{array}$ \\
\hline Parâmetro $\sigma 2$ & & & & $\begin{array}{c}23,592 \\
(2,236)\end{array}$ & $\begin{array}{c}15,077 \\
(0,917)\end{array}$ & $\begin{array}{l}7,881 \\
(0,210)\end{array}$ \\
\hline Observações & 19.353 & 19.353 & 19.353 & 19.353 & 19.353 & 19.353 \\
\hline
\end{tabular}



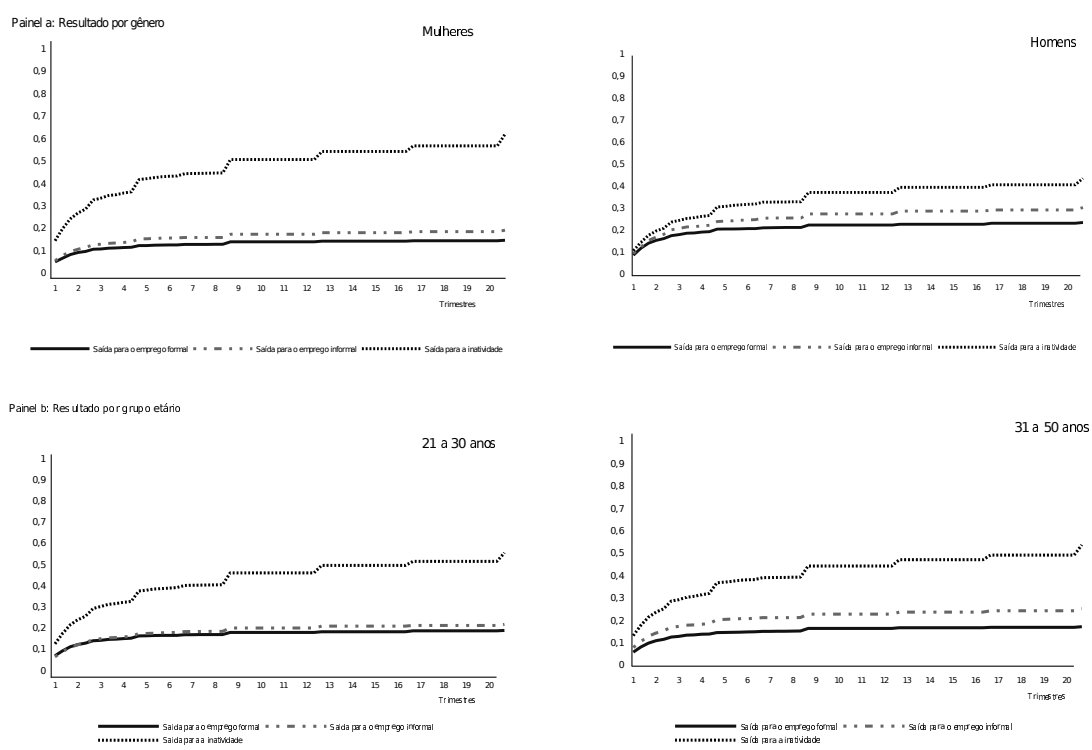

Painel c: Resultado por es colaridade
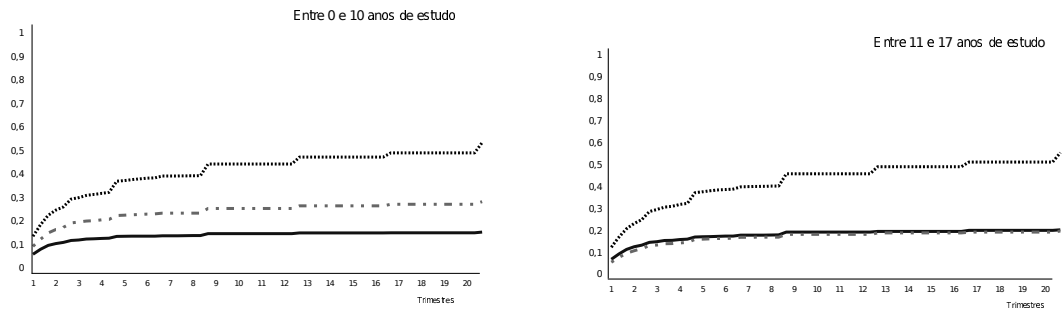

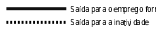

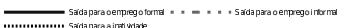

Figura A.1: Função de incidência acumulada 